\title{
The Metabolic Syndrome and Non-Alcoholic Fatty Liver Disease in Children
}

\author{
Shikha S. Sundaram, MD MSCI ${ }^{1}$, Phil Zeitler, MD, PHD $^{2}$, and Kristen Nadeau, MD $^{2}$ \\ ${ }^{1}$ Division of Gastroenterology, Hepatology and Nutrition, Department of Pediatrics, University of \\ Colorado Denver \\ 2 Division of Endocrinology, Department of Pediatrics, University of Colorado Denver
}

\begin{abstract}
Purpose of review-Nonalcoholic fatty liver disease (NAFLD) is increasingly prevalent in pediatric age individuals, in parallel with increasing obesity, and can lead to liver inflammation, fibrosis and even cirrhosis. NAFLD appears tightly linked with features of the metabolic syndrome (MetS). This review aims to review the clinical presentation, laboratory and pathologic assessment, and treatment of NAFLD, with a focus on its relationship with the MetS.
\end{abstract}

Recent findings-NAFLD occurs with a high prevalence and severity in obese, insulin-resistant adolescents, especially Hispanic males. Pediatric NAFLD may improve with lifestyle therapy and agents that improve insulin sensitivity. In youth, NAFLD appears tightly correlated with components of the MetS, especially visceral fat, which appears to predict fibrosis as well as liver fat. In addition, noninvasive techniques such as transient elastography may help provide data on fibrosis in youth with NAFLD and avoid biopsy.

Summary-The close association between NAFLD and the MetS supports screening for other co-morbidities associated with the MetS. Further research is urgently required to best identify effective therapies to prevent and treat NAFLD, but its close association with MetS argues for a focus on strategies designed to improve insulin resistance and components of the MetS.

\section{Keywords}

metabolic syndrome; insulin resistance; hepatic steatosis; fatty liver disease

\section{Introduction}

As obesity in the pediatric age group increases, nonalcoholic fatty liver disease (NAFLD) has become increasingly prevalent and can lead to liver inflammation, fibrosis and even cirrhosis. NAFLD is rapidly becoming the most common cause of liver disease in children and adolescents. NAFLD is strongly associated with features of the metabolic syndrome (MetS) and can be viewed as the hepatic manifestation of insulin resistance. In this review, we will discuss the clinical presentation, laboratory and pathologic assessment, and treatment of NAFLD, with a focus on its relationship with the MetS.

Address Correspondence to: Phil Zeitler MD, PhD, Division of Endocrinology B265, 13123 East $16^{\text {th }}$ Avenue, Aurora CO 80045 , Tel: 720-777-6128, Fax: 720-777-7301, phil.zeitler@ucdenver.edu. 


\section{The Metabolic Syndrome}

The metabolic syndrome (MetS) encompasses a group of factors that together confers an increased risk of cardiovascular disease and is associated with insulin resistance (IR) and type 2 diabetes mellitus (T2D). Although standard definitions of MetS exist in adults, there is currently no uniformly accepted pediatric definition. The most commonly used definition was modified from the National Cholesterol Education Program (NCEP), Adult Treatment Panel III ${ }^{1}$, where individuals must have at least 3 of the following criteria: elevated blood pressure, low HDL cholesterol, high triglycerides, high fasting glucose level, and abdominal obesity. NHANES data from 1988-1994 showed a 4\% prevalence of MetS in adolescents age $12-19$, which increased to $29 \%$ if limited to obese adolescents ${ }^{1}$. A more recent analysis of NHANES data from 1999-2002 showed a MetS prevalence of 7.8\% in overweight and $44 \%$ in obese adolescents ${ }^{2}$. A study of 6,700 children ages 5-17 years, demonstrated that $39 \%$ had MetS, defined as obesity plus at least 2 additional MetS components. If only subjects with a BMI $>99 \%$ were included, MetS prevalence increased to $59 \%$. Recently, Lee et al. found MetS was present in $24-51 \%$ of 251 obese youth, depending on the MetS criteria used ${ }^{3}$.

\section{Non-Alcoholic Fatty Liver Disease (NAFLD)}

Non-alcoholic fatty liver disease (NAFLD) is a clinicopathologic condition characterized by abnormal lipid deposition in hepatocytes (steatosis) in the absence of excess alcohol intake and is widely recognized as the most frequent etiology of chronically elevated aminotransferases among adults and children in the United States ${ }^{4-6}$. NAFLD comprises a spectrum of diseases, ranging from simple hepatic steatosis to steatosis in association with necro-inflammation and fibrosis (non-alcoholic steatohepatitis, NASH) to cirrhosis ${ }^{7}$. Progression of NAFLD can cause liver failure and portal hypertension, leading to the need for liver transplant, even in adolescents. Indeed, NAFLD is rapidly becoming the most common etiology for liver failure in the US. NALFD also increases the risk of liver cancer.

Recent studies suggest that hepatic steatosis is present in greater than $60 \%$ of obese and $90 \%$ of morbidly obese adults 8,9 . Progression to NASH occurs in $19 \%$ of obese, and nearly $50 \%$ of morbidly obese adults, with subsequent progression to liver fibrosis and cirrhosis in approximately $30 \%^{8-11}$. The exact prevalence of pediatric NAFLD is unknown. The prevalence of elevated alanine aminotransferase (ALT) in obese youth is reported as 10$14 \%$ in U.S. adolescents, $24 \%$ in Hispanic youth, $25 \%$ in Italian youth, $24 \%$ in Japanese youth and $48 \%$ in youth with $\mathrm{T} 2 \mathrm{D}^{4}, 12-17$. However, these figures likely underestimate the true pediatric prevalence, which may be present despite normal serum aminotransferase levels. Prevalence estimates of $18-53 \%$ have been derived using ultrasound and MRI. Current imaging techniques, however, do not accurately identify all patients with NAFLD ${ }^{18,19}$. A large pediatric autopsy study found a NAFLD prevalence of $9.6 \%$ after adjustment for age, gender, and ethnicity, with $38 \%$ prevalence in obese youth. The prevalence of fatty liver increased from $0.7 \%$ in 2-4 year olds to $17.3 \%$ in $15-19$ year olds $^{20}$. Disturbingly, pediatric reports indicate a higher initial incidence of fibrosis and cirrhosis than in adults ${ }^{21,22}$. A study of obese children with fatty liver and elevated aminotransferases found NASH in $88 \%$ and fibrosis in $71 \%$.

Adult studies of NAFLD support a female preponderance ${ }^{23,24}$. In children, however, the male to female ratio is $2: 113,17,25-28$. Most children are diagnosed between $11.5-13.5$ years of age, likely at the peak of pubertal IR, though increasing preschool obesity may alter this demographic. Hispanic children have a higher prevalence and severity of fatty liver disease and aminotransferase elevation than African Americans, with the prevalence in Caucasians being intermediate ${ }^{14,16,24,25,29}$. Differences in IR, higher visceral fat and 
triglycerides (components of MetS) reported in Hispanics compared to African American youth are possible explanations ${ }^{14,30-32}$.

\section{The Metabolic Syndrome and Non-Alcoholic Fatty Liver Disease}

Although NAFLD is not traditionally part of the MetS definition, it is widely considered the hepatic manifestation of the MetS. NAFLD is associated with obesity, IR, hypertriglyceridemia, and MetS in adults ${ }^{33-35}$. A recent study of children with biopsy proven NAFLD for MetS according to the NCEP guidelines reported that $66 \%$ had MetS. More specifically, 63\% had hypertryiglyceridemia, 45\% low HDL cholesterol, $40 \%$ hypertension, and $10 \%$ impaired glucose tolerance ${ }^{36}$. Moreover, there was an association between the histologic severity of disease and some components of MetS. In addition, a recent study found that increased ALT was associated with developing MetS over 20 years of follow-up ${ }^{37}$. Finally, NAFLD independently increases the relative risk of cardiovascular events ${ }^{29}$.

Waist circumference is a surrogate marker for visceral fat and visceral fat appears tightly correlated with hepatic triglyceride content, elevated ALT, liver inflammation and fibrosis, and African Americans have both lower visceral and hepatic fat content ${ }^{13}, 38-41$. In a pediatric study, every $1 \mathrm{~cm}$ increase in waist circumference was associated with a 1.97 fold (95\% CI 1.06-3.66) increased risk of nonalcoholic fatty liver disease in boys and a 2.08 fold (95\% CI 1.38-3.13) risk in girls ${ }^{42}$. Waist circumference may also independently predict liver fibrosis on biopsy ${ }^{43}$. Serum triglyceride levels correlate positively with hepatic triglyceride content and are lower in African Americans than in other races ${ }^{13,41,44 .}$

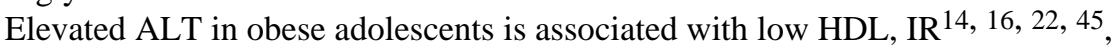
hyperglycemia and T2D ${ }^{17}$. Elevated ALT predicts the development of T2D in adults ${ }^{46}$. IR, particularly T2D, appears to increase the risk of elevated ALT beyond that of obesity alone ${ }^{17,47,48}$.

\section{Overlap of Potential Pathophysiologic Mechanisms: MetS and NAFLD}

The pathogenesis of MetS and NAFLD are incompletely understood. The overlap of potential mechanisms, however, provides insights into their pathogenesis. NAFLD requires an accumulation of hepatocyte lipid in the form of free fatty acids (FFA) and triglycerides. IR suppresses glycogenesis, promotes gluconeogenesis and glycogenolysis and increases FFA release from adipose tissue. Circulating FFA uptake by hepatocytes is unregulated, resulting in increased triglyceride synthesis and impaired FFA oxidation, producing excess hepatocyte lipid ${ }^{49-51}$. Hyperinsulinemia may also increase hepatic triglyceride synthesis by over-stimulating steroid regulatory-element binding protein-1 (SREBP-1c) $)^{52,53}$.

Progression to NASH involves increased hepatocyte susceptibility to oxidative stress, generation of reactive oxygen species, and subsequent lipid peroxidation. By-products of oxidative stress and lipid peroxidation are powerful chemoattractants of neutrophils and stimulate the hepatic stellate cells responsible for fibrosis, along with the release of inflammatory cytokines, including TNF- $\alpha$. TNF- $\alpha$, a pro-inflammatory cytokine, promotes IR, is pro-apoptotic and is important in white blood cell recruitment. It is increased in patients with NAFLD and the MetS. Adiponectin, an anti-inflammatory cytokine, typically inhibits fatty acid uptake, stimulates fatty acid oxidation and lipid export and enhances insulin sensitivity. Adiponectin is decreased in NAFLD and the MetS.

\section{Clinical Presentation of NAFLD}

Most children with NAFLD are asymptomatic and elevated aminotransferases are frequently noted incidentally or after screening for obesity related co-morbidities. Children may also complain of vague right upper quadrant or epigastric pain or fatigue. A thorough history 
often reveals co-morbid conditions related to MetS, including hypertension, T2D, dyslipidemia, obstructive sleep apnea, and polycystic ovarian syndrome. Greater than $90 \%$ of children with NAFLD are obese, with central adiposity ${ }^{54}$. Hepatomegaly, with or without splenomegaly, can be detected in $33-51 \%$ of patients, though central adiposity can make organomegaly difficult to appreciate 22,54 . Acanthosis nigricans may be seen in $36-49 \%$ of NAFLD patients ${ }^{22,54}$.

\section{Laboratory Evaluation of NAFLD}

Serum aminotransferases are usually mild to moderately elevated (less than 1.5 times the upper limit of normal), in NAFLD, but may be higher. The ALT:AST ratio is typically $>1^{22}$, 54-56. However, aminotransferases may remain normal, even with biopsy proven NASH ${ }^{56}$. Total and direct bilirubin levels are typically normal, though GGTP and alkaline phosphatase are mildly elevated in less than $50 \%$ of cases. Abnormal iron indices may occur in adults, with $30 \%$ having high serum ferritin and 6-14\% having elevated transferrin saturation $^{58}$. These indices, however, are not routinely measured in youth, as hemochromatosis is rare in children.

A thorough evaluation and systematic exclusion of other etiologies of liver disease is necessary, including Wilson's disease, alpha-1-antitryspin deficiency, viral hepatitis, autoimmune hepatitis, fatty acid oxidation defects and lipodystrophy. In addition, medications that can induce hepatic steatosis (glucocorticoids, amiodarone, valproate, methotrexate, and synthetic estrogens) should be considered. However, low titers of serum auto-antibodies may occur in up to $3 \%$ of adults with NAFLD; the prevalence in pediatrics is unknown ${ }^{59}$. Finally, alcohol use must be assessed, particularly in adolescents.

In patients experiencing right upper quadrant pain, imaging can help evaluate for hepatic and biliary anatomic abnormalities. Abdominal imaging may also confirm hepatic fatty infiltration consistent with NAFLD. Abdominal ultrasound is relatively inexpensive, noninvasive, and easy to perform. Ultrasound, however, is not typically quantitative, requires a minimum of $30 \%$ hepatic fat for detection ${ }^{60}$, and may be technically challenging to perform with significant central obesity. CT scans have the disadvantage of radiation exposure. MRI, though more costly, is more sensitive in detecting fat and allows for more definitive hepatic fat quantification when performed using the modified Dixon technique or with magnetic resonance spectroscopy 19,61 . Importantly, none of the currently available imaging modalities allow differentiation of benign steatosis from NASH, nor have the ability to grade the severity of inflammation. Newer transient elastography imaging methods may allow detection of fibrosis ${ }^{62}$.

Patients with NAFLD should also be screened for other co-morbid conditions associated with IR and MetS, such as hyperglycemia, dyslipidemia, hypertension, and sleep apnea. Girls should also be screened for menstrual irregularities and hirsutism, symptoms of polycystic ovarian syndrome.

\section{Pathologic Assessment of NAFLD}

The distinction between simple hepatic steatosis and potentially progressive NASH can only be made by liver biopsy, which can assess the presence and extent of necro-inflammation and fibrosis. A liver biopsy should be performed in all patients who do not fit the classic phenotype of NAFLD or have a chronic hepatitis (elevated aminotransferases for greater than 3-6 months). A three-tiered grading and staging system for NASH is now widely used, based on a semi-quantitative evaluation of multiple histologic features ${ }^{7}, 63$. The minimum criteria for NASH are: 1) steatosis, with macrovesicular fat greater than microvesicular fat; 2) mixed, mild lobular inflammation with scattered polymorphonuclear leukocytes and 
mononuclear cells and 3) hepatocyte ballooning that is most apparent near steatotic liver cells.

A unique histologic pattern, deemed Type $2 \mathrm{NASH}$, is reported in pediatrics. In this distinct pattern, inflammation and fibrosis are accentuated in the portal areas, in contrast to the perisinusoidal injury typically observed in adults with NASH (Type $1 \mathrm{NASH})^{28}$. A study of 100 pediatric patients demonstrated Type 2 NASH in 51\%, Type 1 NASH in $17 \%$ and an overlap pattern in $16 \%$ of subjects ${ }^{28}$. A recent Italian study demonstrated that $2.4 \%$ of pediatric NASH patients had Type $1 \mathrm{NASH}, 28.6 \%$ had Type $2 \mathrm{NASH}$, and majority $(52.4 \%)$ had an overlap of the 2 patterns ${ }^{64}$. Therefore, it is likely that a spectrum of disease patterns exist in pediatric NASH.

\section{Treatment of NAFLD/NASH}

Currently, the best therapy for NAFLD is slow, progressive weight loss through dietary modification and exercise. Approximately one pound per week is recommended, as more rapid weight loss may exacerbate NAFLD. The optimal diet for treating NAFLD has not been well established, though the importance of IR suggests that low glycemic diets may be beneficial. Most patients, especially adolescents, however, have little success with lifestyle modification, sparking interest in pharmacologic therapies for NASH. However, studies to date have been limited by lack of placebo control, open-label design, small sample size, and short duration of follow up.

Orlistat, an enteric lipase inhibitor, and sibutramine, a serotonin and noradrenaline reuptake inhibitor, resulted in weight loss and some improvement of NAFLD parameters, but require more rigorous study 65,66 . Studies using rimonabant, a cannabinnoid receptor antagonist, to treat NASH were recently terminated because of safety concerns. Case series of bariatric surgery, both gastric bypass and laparoscopic adjustable gastric banding, in adults with NASH have also shown promise ${ }^{67-69}$. This treatment is controversial in pediatrics, however, and cannot be routinely recommended.

Metformin, a biguanide, is a logical candidate as it improves hepatic IR and is well tolerated in children ${ }^{70}$. Several small adult trials show promising results, and two pediatric studies showed significant improvements in aminotransferases and steatosis ${ }^{14,} 71-75$. A recent metaanalysis demonstrated that metformin leads to normalization of aminotransferases in significantly more patients than dietary modifications alone and also improves steatosis on radiologic imaging ${ }^{76}$. Adequate long-term studies including liver biopsy, however, are lacking. Metformin monotherapy in pediatric NASH is currently undergoing evaluation as part of the National Institutes of Health sponsored NASH Clinical Research Network (CRN) TONIC trial.

Another potentially promising class of drugs are the thiazolidienediones (TZD), including pioglitazone and rosiglitazone. These medications improve IR by acting as selective peroxisome proliferator-activated receptor gamma (PPAR-gamma) agonists. Treatment with TZD results in improved liver biochemistry and histology, though histologic improvements were mainly in steatosis ${ }^{70,77-80}$. These beneficial effects, however, do not persist when the medications are stopped, suggesting that long term treatment may be necessary. Recent concerns regarding increased fractures and possible increases in heart disease in adults may ultimately limit the use of TZDs in pediatrics.

As dyslipidemia is a component of MetS and important in NAFLD physiology, lipid lowering agents may have a potential role in the treatment of NASH. Gemfibrozil improved biochemical parameters in NASH, though clofibrate failed to improve either biochemical or histologic parameters ${ }^{81}, 82$. Several small pilot studies using HMG-CoA reductase inhibitors 
to treat NASH showed improvements in serum aminotransferases and hepatic inflammation ${ }^{83,84}$. However, the potential risk of hepatotoxicity has limited the use of these medications in NAFLD.

The role of oxidative stress in the pathogenesis of NAFLD led to treatment trials of antioxidants. A small open label pilot study of 11 youth with NASH who were treated with Vitamin E for 2-4 months showed normalization of $\mathrm{ALT}^{85}$. Vitamin E monotherapy is also currently being studied thorough the NASH CRN TONIC trial. A small randomized trial of combined Vitamin $\mathrm{E}$ and $\mathrm{C}$ therapy in which all individuals received a tailored diet and increased physical activity failed to demonstrate any added benefit compared to lifestyle interventions alone ${ }^{86}$. A small pilot trial of betaine, a choline metabolite that increases Sadenosylmethionine levels, demonstrated improvements in aminotransferases, steatosis, and necro-inflammation in adult NASH patients ${ }^{87}$. A small open label pilot study of $\mathrm{N}$ acetylcysteine also showed improved aminotransferases in adult NASH patients ${ }^{88}$. Despite significant initial enthusiasm, ursodeoxycholic acid, a cytoprotective bile acid, was ineffective in normalizing aminotransferases or improving histologic parameters in $\mathrm{NASH}^{89}, 90$. Given the central role of TNF- $\alpha$ in the development of necro-inflammation in NASH, pentoxyfilline, a TNF- $\alpha$ inhibitor has also been studied. Two small adult pilot studies demonstrated improvements in aminotransferases ${ }^{91,92}$. An open label follow-up study of 9 subjects with biopsy proven NASH also showed histologic improvement ${ }^{93}$. A significant number of patients, however, experienced nausea, rendering this drug difficult to tolerate.

\section{Conclusions}

The high prevalence of NAFLD and elevated aminotransferases in obese adolescents with IR, and the implications for their health is concerning. The close associations between NAFLD and MetS support screening for other co-morbidities associated with MetS. While maintaining a healthy weight and exercising regularly are still critical to improving the health of IR teens, behavioral interventions are especially difficult amidst the chaos of adolescence. Therefore, additional therapies to improve liver health are urgently needed in this high-risk population. Treatment strategies designed to improve IR and components of MetS hold significant promise.

\section{References}

1. Cook S, Weitzman M, Auinger P, et al. Prevalence of a metabolic syndrome phenotype in adolescents: findings from the third National Health and Nutrition Examination Survey, 1988-1994. Arch Pediatr Adolesc Med. 2003; 157:821-7. [PubMed: 12912790]

2. Cook S, Auinger P, Li C, Ford ES. Metabolic syndrome rates in United States adolescents, from the National Health and Nutrition Examination Survey, 1999-2002. J Pediatr. 2008; 152:165-70. [PubMed: 18206683]

3. Lee $S$, et al. Comparison of different definitions of pediatric metabolic syndrome: relation to abdominal adiposity, insulin resistance, adiponectin, and inflammatory biomarkers. J Pediatr. 2008; 152(2):177-84. [PubMed: 18206686]

4. Franzese A, Vajro P, Argenziano A, et al. Liver involvement in obese children. Ultrasonography and liver enzyme levels at diagnosis and during follow-up in an Italian population. Dig Dis Sci. 1997; 42:1428-32. [PubMed: 9246041]

5. Clark JM, Brancati FL, Diehl AM. The prevalence and etiology of elevated aminotransferase levels in the United States. Am J Gastroenterol. 2003; 98:960-7. [PubMed: 12809815]

6. Daniel S, Ben-Menachem T, Vasudevan G, et al. Prospective evaluation of unexplained chronic liver transaminase abnormalities in asymptomatic and symptomatic patients. Am J Gastroenterol. 1999; 94:3010-4. [PubMed: 10520861] 
7. Brunt EM. Nonalcoholic steatohepatitis: definition and pathology. Semin Liver Dis. 2001; 21:3-16. [PubMed: 11296695]

8. Silverman JF, O'Brien KF, Long S, et al. Liver pathology in morbidly obese patients with and without diabetes. Am J Gastroenterol. 1990; 85:1349-55. [PubMed: 2220728]

9. Wanless IR, Lentz JS. Fatty liver hepatitis (steatohepatitis) and obesity: an autopsy study with analysis of risk factors. Hepatology. 1990; 12:1106-10. [PubMed: 2227807]

10. Matteoni CA, Younossi ZM, Gramlich T, et al. Nonalcoholic fatty liver disease: a spectrum of clinical and pathological severity. Gastroenterology. 1999; 116:1413-9. [PubMed: 10348825]

11. Falck-Ytter Y, Younossi ZM, Marchesini G. Clinical features and natural history of nonalcoholic steatosis syndromes. Semin Liver Dis. 2001; 21:17-26. [PubMed: 11296693]

12. Strauss RS, Barlow SE, Dietz WH. Prevalence of abnormal serum aminotransferase values in overweight and obese adolescents. J Pediatr. 2000; 136:727-33. [PubMed: 10839867]

13. Burgert TS, Taksali SE, Dziura J, et al. Alanine aminotransferase levels and fatty liver in childhood obesity: associations with insulin resistance, adiponectin, and visceral fat. J Clin Endocrinol Metab. 2006; 91:4287-94. [PubMed: 16912127]

14*. Nadeau KJ, Ehlers LB, Zeitler PS, Love-Osborne K. Treatment of non-alcoholic fatty liver disease with metformin versus lifestyle intervention in insulin-resistant adolescents. Pediatr Diabetes. 2008 Fatty liver was more common in male and Hispanic youth and youth who were more insulin resistant. Treatment with metformin improved fatty liver prevalence, severity, and fasting insulin compared to placebo.

15. Quiros-Tejeira RE, Rivera CA, Ziba TT, et al. Risk for nonalcoholic fatty liver disease in Hispanic youth with BMI > or $=95$ th percentile. J Pediatr Gastroenterol Nutr. 2007; 44:228-36. [PubMed: 17255837]

16. Kawasaki T, Hashimoto N, Kikuchi T, et al. The relationship between fatty liver and hyperinsulinemia in obese Japanese children. J Pediatr Gastroenterol Nutr. 1997; 24:317-21. [PubMed: 9138179]

17. Nadeau KJ, Klingensmith G, Zeitler P. Type 2 diabetes in children is frequently associated with elevated alanine aminotransferase. J Pediatr Gastroenterol Nutr. 2005; 41:94-8. [PubMed: 15990637]

18. Guzzaloni G, Grugni G, Minocci A, et al. Liver steatosis in juvenile obesity: correlations with lipid profile, hepatic biochemical parameters and glycemic and insulinemic responses to an oral glucose tolerance test. Int J Obes Relat Metab Disord. 2000; 24:772-6. [PubMed: 10878685]

19. Fishbein MH, Gardner KG, Potter CJ, et al. Introduction of fast MR imaging in the assessment of hepatic steatosis. Magn Reson Imaging. 1997; 15:287-93. [PubMed: 9201675]

20. Schwimmer JB, Deutsch R, Kahen T, et al. Prevalence of fatty liver in children and adolescents. Pediatrics. 2006; 118:1388-93. [PubMed: 17015527]

21. Nadeau K, Klingensmith G, Sokol RJ. Case report: nonalcoholic steatohepatitis in a teenage girl with type 2 diabetes. Curr Opin Pediatr. 2003; 15:127-31. [PubMed: 12544284]

22. Rashid M, Roberts EA. Nonalcoholic steatohepatitis in children. J Pediatr Gastroenterol Nutr. 2000; 30:48-53. [PubMed: 10630439]

23. Ludwig J, Viggiano TR, McGill DB, Oh BJ. Nonalcoholic steatohepatitis: Mayo Clinic experiences with a hitherto unnamed disease. Mayo Clin Proc. 1980; 55:434-8. [PubMed: 7382552]

24. Diehl AM, Goodman Z, Ishak KG. Alcohollike liver disease in nonalcoholics. A clinical and histologic comparison with alcohol-induced liver injury. Gastroenterology. 1988; 95:1056-62. [PubMed: 3410220]

25. Fraser A, Longnecker MP, Lawlor DA. Prevalence of elevated alanine aminotransferase among US adolescents and associated factors: NHANES 1999-2004. Gastroenterology. 2007; 133:1814-20. [PubMed: 18054554]

26. Sartorio A, Del Col A, Agosti F, et al. Predictors of non-alcoholic fatty liver disease in obese children. Eur J Clin Nutr. 2007; 61:877-83. [PubMed: 17151586]

27. Kinugasa A, Tsunamoto K, Furukawa N, et al. Fatty liver and its fibrous changes found in simple obesity of children. J Pediatr Gastroenterol Nutr. 1984; 3:408-14. [PubMed: 6737186] 
28. Schwimmer JB, Behling C, Newbury R, et al. Histopathology of pediatric nonalcoholic fatty liver disease. Hepatology. 2005; 42:641-9. [PubMed: 16116629]

29. Targher G, Bertolini L, Poli F, et al. Nonalcoholic fatty liver disease and risk of future cardiovascular events among type 2 diabetic patients. Diabetes. 2005; 54:3541-6. [PubMed: 16306373]

30. Ellis KJ. Body composition of a young, multiethnic, male population. Am J Clin Nutr. 1997; 66:1323-31. [PubMed: 9394682]

31. Ellis KJ, Abrams SA, Wong WW. Body composition of a young, multiethnic female population. Am J Clin Nutr. 1997; 65:724-31. [PubMed: 9062521]

32. Bacha F, Saad R, Gungor N, et al. Obesity, regional fat distribution, and syndrome X in obese black versus white adolescents: race differential in diabetogenic and atherogenic risk factors. $\mathrm{J}$ Clin Endocrinol Metab. 2003; 88:2534-40. [PubMed: 12788850]

33. Marchesini G, Brizi M, Morselli-Labate AM, et al. Association of nonalcoholic fatty liver disease with insulin resistance. Am J Med. 1999; 107:450-5. [PubMed: 10569299]

34. Marchesini G, Brizi M, Bianchi G, et al. Nonalcoholic fatty liver disease: a feature of the metabolic syndrome. Diabetes. 2001; 50:1844-50. [PubMed: 11473047]

35. Fraser A, Ebrahim S, Smith GD, Lawlor DA. A comparison of associations of alanine aminotransferase and gamma-glutamyltransferase with fasting glucose, fasting insulin, and glycated hemoglobin in women with and without diabetes. Hepatology. 2007; 46:158-65. [PubMed: 17596883]

36**. Manco M, Marcellini M, Devito R, et al. Metabolic syndrome and liver histology in paediatric non-alcoholic steatohepatitis. Int J Obes (Lond). 2008; 32:381-7. Among a cohort of 120 children (3-18 years) with NAFLD or NASH, the majority had the metabolic syndrome, and levels of aminotransferases, steatosis and fibrosis were higher in subjects with the metabolic syndrome. Thus, presence of the metabolic syndrome or clinical and biochemical variables associated with the syndrome seems to predict features of NASH in pediatric fatty liver disease, and may help direct which youth with NAFLD should undergo biopsy. [PubMed: 18087267]

37*. Goessling W, Massaro JM, Vasan RS, et al. Aminotransferase levels and 20-year risk of metabolic syndrome, diabetes, and cardiovascular disease. Gastroenterology. 2008; 135:1935-44. 1944 e1. Among adults from the Framingham Offspring Heart Study, as ALT levels increased, even within the normal range, the odds of developing metabolic syndrome, diabetes, and cardiovascular disease increased, indicating that subjects with increased ALT should be watched more closely and potentially treated more aggressively for these disorders. [PubMed: 19010326]

38. Fishbein MH, Mogren C, Gleason T, Stevens WR. Relationship of hepatic steatosis to adipose tissue distribution in pediatric nonalcoholic fatty liver disease. J Pediatr Gastroenterol Nutr. 2006; 42:83-8. [PubMed: 16385259]

39* . van der Poorten D, Milner KL, Hui J, et al. Visceral fat: a key mediator of steatohepatitis in metabolic liver disease. Hepatology. 2008; 48:449-57. Visceral fat is directly associated with liver inflammation and fibrosis in adults, independent of insulin resistance and hepatic steatosis. Visceral fat may be a central target for future interventions in nonalcoholic steatohepatitis. [PubMed: 18627003]

40. Bahl M, Qayyum A, Westphalen AC, et al. Liver steatosis: investigation of opposed-phase T1weighted liver MR signal intensity loss and visceral fat measurement as biomarkers. Radiology. 2008; 249:160-6. [PubMed: 18796674]

$41 * *$. Guerrero R, Vega GL, Grundy SM, Browning JD. Ethnic differences in hepatic steatosis: An insulin resistance paradox? Hepatology. 2008 Using proton magnetic resonance (MR) spectroscopy, dual-energy x-ray absorptiometry, and multislice abdominal MR imaging AfricanAmericans had less intraperitoneal (IP) fat, less hepatic fat, more lower extremity fat, and lower triglycerides than their Hispanic and Caucasian counterparts but were equally insulin resistant. Controlling for intraperitoneal fat nearly abolished the differences in hepatic triglyceride. Therefore, differences in hepatic fat between ethnic groups is linked to differences in visceral fat, and African Americans may have a very different phenotype of insulin resistance than Hispanic or Caucasians.

42**. Damaso AR, do Prado WL, de Piano A, et al. Relationship between nonalcoholic fatty liver disease prevalence and visceral fat in obese adolescents. Dig Liver Dis. 2008; 40:132-9. In 
obese, postpubertal adolescents, increasing visceral fat was associated with increasing risk of NAFLD, demonstrating that visceral fat and NAFLD are linked in adolescents as well as in adults. [PubMed: 18082476]

43**. Manco M, Bedogni G, Marcellini M, et al. Waist circumference correlates with liver fibrosis in children with non-alcoholic steatohepatitis. Gut. 2008; 57:1283-7. In children with NAFLD (319 years), 67\% had liver fibrosis, although mainly of a low degree. In multivariable analysis, waist circumference was the only metabolic syndrome component to be associated with liver fibrosis. Therefore, not only is abdominal fat associated with NAFLD, but also with increased risk of fibrosis. [PubMed: 18218674]

44. de Piano A, Prado WL, Caranti DA, et al. Metabolic and nutritional profile of obese adolescents with nonalcoholic fatty liver disease. J Pediatr Gastroenterol Nutr. 2007; 44:446-52. [PubMed: 17414142]

45. Nadeau K, Klingensmith GK, Zeitler PS. Nonalcoholic fatty liver disease in pediatric type 2 diabetics. Journal of Investigative Medicine. 2003; 51(Sup2):S378.

46. Sattar N, Scherbakova O, Ford I, et al. Elevated alanine aminotransferase predicts new-onset type 2 diabetes independently of classical risk factors, metabolic syndrome, and C-reactive protein in the west of Scotland coronary prevention study. Diabetes. 2004; 53:2855-60. [PubMed: 15504965]

47. Salmela PI, Sotaniemi EA, Niemi M, Maentausta O. Liver function tests in diabetic patients. Diabetes Care. 1984; 7:248-54. [PubMed: 6734394]

48. Silverman JF, Pories WJ, Caro JF. Liver pathology in diabetes mellitus and morbid obesity. Clinical, pathological, and biochemical considerations. Pathol Annu. 1989; 24(Pt 1):275-302. [PubMed: 2654841]

49. Harrison SA, Neuschwander-Tetri BA. Nonalcoholic fatty liver disease and nonalcoholic steatohepatitis. Clin Liver Dis. 2004; 8:861-79. ix. [PubMed: 15464659]

50. Chitturi S, Farrell GC. Etiopathogenesis of nonalcoholic steatohepatitis. Semin Liver Dis. 2001; 21:27-41. [PubMed: 11296694]

51. Saltiel AR, Kahn CR. Insulin signalling and the regulation of glucose and lipid metabolism. Nature. 2001; 414:799-806. [PubMed: 11742412]

52. Fleischmann M, Iynedijan PB. Regulation of sterol regulatory-element binding protein 1 gene expression in liver: role of insulin and protein kinase B/cAkt. Biochem J. 2000; 349:13-17. [PubMed: 10861205]

53. Shimomura I. Insulin selectively increases SREBP-1c mRNA in livers of rats with streptozotocininduced diabetes. Proc Nat Acad Sci. 1999; 96:13656-13661. [PubMed: 10570128]

54. Schwimmer JB, Deutsch R, Rauch JB, et al. Obesity, insulin resistance, and other clinicopathological correlates of pediatric nonalcoholic fatty liver disease. J Pediatr. 2003; 143:500-5. [PubMed: 14571229]

55. Baldridge AD, Perez-Atayde AR, Graeme-Cook F, et al. Idiopathic steatohepatitis in childhood: a multicenter retrospective study. J Pediatr. 1995; 127:700-4. [PubMed: 7472819]

56. Manton ND, Lipsett J, Moore DJ, et al. Non-alcoholic steatohepatitis in children and adolescents. Med J Aust. 2000; 173:476-9. [PubMed: 11149304]

57. Lavine JE, Schwimmer JB. Nonalcoholic fatty liver disease in the pediatric population. Clin Liver Dis. 2004; 8:549-58. viii-ix. [PubMed: 15331063]

58. Bugianesi E, Manzini P, D'Antico S, et al. Relative contribution of iron burden, HFE mutations, and insulin resistance to fibrosis in nonalcoholic fatty liver. Hepatology. 2004; 39:179-87. [PubMed: 14752836]

59. Cotler SJ, Kanji K, Keshavarzian A, et al. Prevalence and significance of autoantibodies in patients with non-alcoholic steatohepatitis. J Clin Gastroenterol. 2004; 38:801-4. [PubMed: 15365409]

60. Saadeh S, Younossi ZM, Remer EM, et al. The utility of radiological imaging in nonalcoholic fatty liver disease. Gastroenterology. 2002; 123:745-50. [PubMed: 12198701]

61. Fishbein MH, Stevens WR. Rapid MRI using a modified Dixon technique: a non-invasive and effective method for detection and monitoring of fatty metamorphosis of the liver. Pediatr Radiol. 2001; 31:806-9. [PubMed: 11692239]

$62 * *$. Nobili V, Vizzutti F, Arena U, et al. Accuracy and reproducibility of transient elastography for the diagnosis of fibrosis in pediatric nonalcoholic steatohepatitis. Hepatology. 2008; 48:442-8. 
This study of pediatric patients with nonalcoholic steatohepatitis showed that noninvasive transient elastography is an accurate and reproducible methodology to identify pediatric subjects without fibrosis or significant fibrosis, or with advanced fibrosis. Such techniques may help to reduce the number of biopsies required in youth with NAFLD. [PubMed: 18563842]

63. Brunt EM, Janney CG, Di Bisceglie AM, et al. Nonalcoholic steatohepatitis: a proposal for grading and staging the histological lesions. Am J Gastroenterol. 1999; 94:2467-74. [PubMed: 10484010]

64. Nobili V, Marcellini M, Devito R, et al. NAFLD in children: a prospective clinical-pathological study and effect of lifestyle advice. Hepatology. 2006; 44:458-65. [PubMed: 16871574]

65. Harrison SA, Ramrakhiani S, Brunt EM, et al. Orlistat in the treatment of NASH: a case series. Am J Gastroenterol. 2003; 98:926-30. [PubMed: 12738478]

66. Sabuncu T, Nazligul Y, Karaoglanoglu M, et al. The effects of sibutramine and orlistat on the ultrasonographic findings, insulin resistance and liver enzyme levels in obese patients with nonalcoholic steatohepatitis. Rom J Gastroenterol. 2003; 12:189-92. [PubMed: 14502318]

67. Dixon JB, Bhathal PS, Hughes NR, O’Brien PE. Nonalcoholic fatty liver disease: Improvement in liver histological analysis with weight loss. Hepatology. 2004; 39:1647-54. [PubMed: 15185306]

68. Kral JG, Thung SN, Biron S, et al. Effects of surgical treatment of the metabolic syndrome on liver fibrosis and cirrhosis. Surgery. 2004; 135:48-58. [PubMed: 14694300]

69. Luyckx FH, Desaive C, Thiry A, et al. Liver abnormalities in severely obese subjects: effect of drastic weight loss after gastroplasty. Int J Obes Relat Metab Disord. 1998; 22:222-6. [PubMed: 9539189]

70. Tiikkainen M, Hakkinen AM, Korsheninnikova E, et al. Effects of rosiglitazone and metformin on liver fat content, hepatic insulin resistance, insulin clearance, and gene expression in adipose tissue in patients with type 2 diabetes. Diabetes. 2004; 53:2169-76. [PubMed: 15277403]

71. Marchesini G, Brizi M, Bianchi G, et al. Metformin in non-alcoholic steatohepatitis. Lancet. 2001; 358:893-4. [PubMed: 11567710]

72. Nair S, Diehl AM, Wiseman M, et al. Metformin in the treatment of non-alcoholic steatohepatitis: a pilot open label trial. Aliment Pharmacol Ther. 2004; 20:23-8. [PubMed: 15225167]

73. Bugianesi E, Gentilcore E, Manini R, et al. A randomized controlled trial of metformin versus vitamin E or prescriptive diet in nonalcoholic fatty liver disease. Am J Gastroenterol. 2005; 100:1082-90. [PubMed: 15842582]

74. Uygun A, Kadayifci A, Isik AT, et al. Metformin in the treatment of patients with non-alcoholic steatohepatitis. Aliment Pharmacol Ther. 2004; 19:537-44. [PubMed: 14987322]

75. Schwimmer JB, Middleton MS, Deutsch R, Lavine JE. A phase 2 clinical trial of metformin as a treatment for non-diabetic paediatric non-alcoholic steatohepatitis. Aliment Pharmacol Ther. 2005; 21:871-9. [PubMed: 15801922]

76. Angelico F, Burattin M, Alessandri C, et al. Drugs improving insulin resistance for non-alcoholic fatty liver disease and/or non-alcoholic steatohepatitis. Cochrane Database Syst Rev. 2007:CD005166. [PubMed: 17253544]

77. Promrat K, Lutchman G, Uwaifo GI, et al. A Piolot Study of Pioglitazone Treatment for Nonalcoholic Steatohepatitis. Hepatology. 2004; 39:188-196. [PubMed: 14752837]

78. Neuschwander-Tetri BA, Brunt EM, Wehmeier KR, et al. Improved nonalcoholic steatohepatitis after 48 weeks of treatment with the PPAR-gamma ligand rosiglitazone. Hepatology. 2003; 38:1008-17. [PubMed: 14512888]

79. Mayerson AB, Hundal RS, Dufour S, et al. The effects of rosiglitazone on insulin sensitivity, lipolysis, and hepatic and skeletal muscle triglyceride content in patients with type 2 diabetes. Diabetes. 2002; 51:797-802. [PubMed: 11872682]

80. Bajaj M, Suraamornkul S, Pratipanawatr T, et al. Pioglitazone reduces hepatic fat content and augments splanchnic glucose uptake in patients with type 2 diabetes. Diabetes. 2003; 52:1364-70. [PubMed: 12765945]

81. Basaranoglu M, Acbay O, Sonsuz A. A controlled trial of gemfibrozil in the treatment of patients with nonalcoholic steatohepatitis. J Hepatol. 1999; 31:384. [PubMed: 10453959]

82. Laurin J, Lindor KD, Crippin JS, et al. Ursodeoxycholic acid or clofibrate in the treatment of nonalcohol-induced steatohepatitis: a pilot study. Hepatology. 1996; 23:1464-7. [PubMed: 8675165] 
83. Rallidis LS, Drakoulis CK, Parasi AS. Pravastatin in patients with nonalcoholic steatohepatitis: results of a pilot study. Atherosclerosis. 2004; 174:193-6. [PubMed: 15135271]

84. Gomez-Dominguez E, Gisbert JP, Moreno-Monteagudo JA, et al. A pilot study of atorvastatin treatment in dyslipemid, non-alcoholic fatty liver patients. Aliment Pharmacol Ther. 2006; 23:1643-7. [PubMed: 16696815]

85. Lavine JE. Vitamin E treatment of nonalcoholic steatohepatitis in children: a pilot study. J Pediatr. 2000; 136:734-8. [PubMed: 10839868]

86** . Nobili V, Manco M, Devito R, et al. Lifestyle intervention and antioxidant therapy in children with nonalcoholic fatty liver disease: a randomized, controlled trial. Hepatology. 2008; 48:11928. Youth (age 5.7-18.8 years) were treated with lifestyle intervention plus either alphatocopherol $600 \mathrm{IU} /$ day and ascorbic acid $500 \mathrm{mg} /$ day or placebo for 24 months. Weight loss resulted in significant improvement in the grade of steatosis, lobular inflammation, and hepatocyte ballooning, and in the NAFLD activity score by liver biopsy, with no differences between groups. This study showed that a lifestyle intervention with diet and increased physical activity is associated with a significant improvement in liver histology and laboratory abnormalities in pediatric NAFLD. Antioxidant therapy provided no additional benefit. This study is unique in being one of the few pediatric studies with repeat liver biopsy findings. [PubMed: 18537181]

87. Abdelmalek MF, Angulo P, Jorgensen RA, et al. Betaine, a promising new agent for patients with nonalcoholic steatohepatitis: results of a pilot study. Am J Gastroenterol. 2001; 96:2711-7. [PubMed: 11569700]

88. Gulbahar OKZ, Ersoz G. Treatment of non-alcoholic steatohepatitis with N-acetylcysteine. Gastroenterology. 2000; 118:A1444.

89. Lindor KD, Kowdley KV, Heathcote EJ, et al. Ursodeoxycholic acid for treatment of nonalcoholic steatohepatitis: results of a randomized trial. Hepatology. 2004; 39:770-8. [PubMed: 14999696]

90. Vajro P, Franzese A, Valerio G, et al. Lack of efficacy of ursodeoxycholic acid for the treatment of liver abnormalities in obese children. J Pediatr. 2000; 136:739-43. [PubMed: 10839869]

91. Adams LA, Zein CO, Angulo P, Lindor KD. A pilot trial of pentoxifylline in nonalcoholic steatohepatitis. Am J Gastroenterol. 2004; 99:2365-8. [PubMed: 15571584]

92. Satapathy SK, Garg S, Chauhan R, et al. Beneficial effects of tumor necrosis factor-alpha inhibition by pentoxifylline on clinical, biochemical, and metabolic parameters of patients with nonalcoholic steatohepatitis. Am J Gastroenterol. 2004; 99:1946-52. [PubMed: 15447754]

93. Satapathy SK, Sakhuja P, Malhotra V, et al. Beneficial effects of pentoxifylline on hepatic steatosis, fibrosis and necroinflammation in patients with non-alcoholic steatohepatitis. J Gastroenterol Hepatol. 2007; 22:634-8. [PubMed: 17444848] 